\section{Pseudoexfoliation as a risk factor for peripheral vascular disease: a case- control study}

MR Praveen', SK Shah', AR Vasavada', RP Diwan', SM Shah', BR Zumkhawala' and R Thomas ${ }^{2,3}$

\begin{abstract}
${ }^{1}$ lladevi Cataract \& IOL Research Center, Raghudeep Eye Clinic, Ahmedabad, India
\end{abstract}

${ }^{2}$ Queensland Eye Institute, South Brisbane, Queensland, Australia

\section{${ }^{3}$ Prevent Blindness}

Foundation,

Wooloongabba,

Queensland, Australia

Correspondence:

AR Vasavada, lladevi Cataract \& IOL Research

Center, Raghudeep Eye

Clinic, Gurukul Road, Memnagar, Ahmedabad 380052, India.

Tel: + 91792749 2303/27

49 0909;

Fax: +917927411200.

E-mail: icirc@

abhayvasavada.com

Received: 22 March 2010 Accepted in revised form: 28 September 2010; Published online: 3

December 2010

Presented in part as a paper at American Academy of Ophthalmology, 11-14 November 2006; Las Vegas, NV, USA.

\begin{abstract}
Aim To determine the association between pseudoexfoliation (PEX) and peripheral vascular disease (PVD) among age-related cataract.

Setting Iladevi Cataract and IOL Research Center, Ahmedabad, India.

Material and methods An observational agematched case-control study of $\mathbf{1 6 0}$ patients over 60 years of age with age-related cataract. A total of 40 subjects with PEX (cases) were compared with 120 subjects with cataract but without PEX (controls). A detailed medical history, including hypertension, diabetes mellitus, cerebrovascular stroke and ischaemic heart disease, was recorded. Ankle brachial index (ABI) was used to determine the risk of PVD among age-related cataract patients. Color Doppler imaging was performed on the brachial and dorsalis pedis artery to measure $\mathrm{ABI}$ and detect PVD. Least mean ABI was the main outcome measure, as low ABI indicates higher risk for PVD. The lowest mean ABI was measured for each subject. An ABI ratio of $<0.90$ was considered abnormal. The MannWhitney $U$-test and logistic regression were used for analysis.

Results The lowest mean ABI in the controls was $0.98 \pm 0.03$ ( $\mathrm{SD}$; a range of $0.86-1.08$ ) as compared with $0.88 \pm 0.02$ (SD) among the cases (a range of $0.79-0.92 ; P<0.001$ ). When compared with controls, cases had a lower ABI $(P<0.001)$ irrespective of the presence or absence of systemic illness. On multiple regression analysis adjusting for systemic illness, the presence of PEX increased the odds of a low ABI group 150 times $(P<0.001)$. Conclusion Subjects with cataract and PEX had a significantly lower ABI as compared with controls (cataracts without PEX). PEX is associated with and may be a risk factor for PVD.
\end{abstract}

Eye (2011) 25, 174-179; doi:10.1038/eye.2010.175; published online 3 December 2010

Keywords: pseudoexfoliation; ABI; PVD; cataract;

Pseudoexfoliation (PEX), an age-related disease, with a reported prevalence of $1.8-13.5 \%$ is characterized by the deposition of a typical fibrillar material in the ocular tissues. ${ }^{1,2}$ PEX is linked to glaucoma and has a higher rate of intraoperative and postoperative complications during cataract surgery. ${ }^{3-5}$ Both undilated and dilated slit-lamp biomicroscopy are used during the clinical examination to detect the white dandruff like material on the pupil, lens, and the angle, as well as other signs such as parapupillary transillumination defects. ${ }^{6,7}$ PEX is not only an ocular disease, but also a generalized disorder that involves the abnormal production and/or turnover of extracellular matrix material. ${ }^{6-10}$ Recent investigations have revealed the presence of PEX fibril in several extracellular tissues. ${ }^{9-11}$ These studies have shown that the PEX material is found in many parts of the body such as the eyes, skin, heart, lungs, liver, kidney, gall bladder, blood vessels, optic nerves, and meninges. ${ }^{9-11}$ PEX has also been shown to affect smaller vessels rather than the major ones. ${ }^{11}$ With studies hypothesizing that hypoxia has an important contributory role in the development of PEX, there is the question of a possible link between the presence of ocular PEX and vascular disease.,12

The presence of peripheral vascular disease (PVD) indicates widespread atherosclerosis in other vascular territories, such the coronary, carotid, and cardiovascular arteries. ${ }^{13,14}$ There is a fourfold to sixfold increase in cardiovascular mortality rate among patients with objectively documented PVD as compared with healthy, age-matched individuals. ${ }^{14}$ However, to the best 
of our knowledge, the association between ocular PEX and PVD has not been reported.

Ankle brachial index (ABI) is used to diagnose PVD of the extremities in symptomatic patients, as well as to assess vascular risk in asymptomatic ones. ${ }^{13,14} \mathrm{ABI}$ has a high sensitivity (90\%) and specificity (98\%) for detecting $\geq 50 \%$ stenosis in the leg arteries. ${ }^{15}$ A low ABI indicates both the presence of flow-limiting atherosclerosis in a peripheral artery and generalized atherosclerosis. ${ }^{13,14}$ Further, it has been established that there is a higher prevalence of low $A B I$ in patients with systemic disorders like diabetes mellitus (DM), hypertension (HT), smoking, angina, acute myocardial infarction, and transient ischaemic attacks. ${ }^{13-15}$ To the best of our knowledge, this is the first reported association between PEX and low ABI, which indicates the presence of a PVD. The aim of this case-control study is to determine the association between ocular PEX and PVD using the $\mathrm{ABI}$ values derived from color Doppler imaging (CDI) measurements.

\section{Material and methods}

An observational case-control study was undertaken at Iladevi Cataract and IOL Research Center, Ahmedabad, India, from 1 June 2006 to 30 June 2007. In all, 160 consecutive patients over 60 years of age with senile cataract were recruited. Subjects with PEX were designated as cases ( $n=40$ patients) and those without PEX constituted the controls ( $n=120$ patients). The diagnosis of PEX required the presence of a classic (late stage) PEX deposition pattern on the anterior lens capsule as a central grey disc, mid-peripheral clear ring, and peripheral grey rim. ${ }^{1}$ Eyes with a history of intraocular surgery, ocular trauma, uveitis, prophylactic laser photocoagulation, or cryo treatment were excluded.

All the patients were evaluated on an outpatient basis. A detailed medical history was recorded, including that of HT, DM, cerebrovascular stroke, and ischaemic heart disease (IHD). While the pupils were dilating, the participants were subjected to an interview. During the interview, the examiner (MRP) asked participants whether they had ever been told by their doctors that they suffered from any of the following: angina or a heart attack, IHD a transient ischaemic attack, a cerebrovascular stroke, DM, HT and if so, whether they were taking any medication. The examiner also asked participants whether they had undergone bypass surgery or angioplasty. DM was defined as a previous history of diabetes treated with insulin, oral hypoglycemic agents, or diet control. Newly diagnosed diabetes was defined as no previous medical history of diabetes in the presence of elevated glycosylated haemoglobin. A positive history of heart attack, bypass surgery, angioplasty, and angina was considered as cardiovascular disease. HT was defined as a systolic blood pressure of $160 \mathrm{~mm} / \mathrm{Hg}$ or more or a diastolic blood pressure of $90 \mathrm{~mm} / \mathrm{Hg}$ or more at the time of the examination (the measurement was not repeated) or a history of HT and current use of medication for it.

Age-matched controls were from the same geographic region as the cases. We selected subjects from patients examined during the period June 2006-June 2007. The selection of the subjects in the control group was done from a frame of 204 cataract patients seen during the period. When a case with PEX was encountered, five matching patients without PEX were randomly selected over the next 3 days to constitute a frame for a second stage of selection. Once the number reached five for a particular age group, three patients from this frame of five were randomly selected for final inclusion. All the patients underwent a complete ocular examination comprising undilated and dilated slit-lamp biomicroscopy, applanation tonometer, and where possible, a fundus examination. In addition to detecting PEX material, the presence of phacodonesis was specifically recorded.

As PEX is relatively rare, the sample size was calculated on the basis of the mean ABI determined among cataract patients posted for surgery. The mean 'lowest' ABI value (defined later) in controls was found to be 1 with a SD of 0.11 . Assuming the difference in the mean lowest ABI value to be $5 \%$ or more in PEX patients, it was necessary to recruit 40 cases and 120 controls in order to have $80 \%$ power to detect a $5 \%$ difference in $\mathrm{ABI}$ between cases and controls. Although the mean lowest ABI did not vary significantly across age groups in controls, it was decided that controls would be recruited in a ratio of $3: 1$ with appropriate age group matching.

A color Doppler machine Shimadzu SDU 2200 (Shimadzu Corporation, Kyoto, Japan) was used to measure ABI in all the patients by recording the blood pressure in the four limbs when the patients were in a supine position. The Doppler imaging records blood flow, which is superimposed in color on a conventional grey scale ultrasound picture. The color image is used as a guide to detect the blood vessels. The Doppler spectral analysis also allows quantitative assessment of the blood flow velocities within the blood vessels. ABI is the ratio of the ankle to brachial systolic blood pressure and a value of $<0.90$ indicates the presence of flow-limiting arterial disease affecting the limbs. ${ }^{15}$ To calculate ABI, the patient was placed in a supine position and the Doppler ultrasound was used to obtain the brachial and ankle systolic pressure measurements in each arm and in the dorsalis pedis arteries in each ankle. CDI demonstrates simultaneous two-dimensional imaging of anatomic structures and blood flow. All the ultrasound 
examinations were performed by a single radiologist and two or more recordings were made for each side (right and left sides). The radiologist was aware of the study hypothesis but was masked to the ophthalmic diagnosis and also on the systemic disease that is prevalent. The higher systolic pressures obtained in the two arms and ankles were taken. The right and left ABI values were determined by dividing the higher ankle pressure in the right and left legs by the higher arm pressure in either arm. Of the two values of ABI obtained from each patient, the lower value was used to assess increased risk of occurrence of PVD in patients with and without PEX; this was done for both cases and controls. An ABI ratio of $<0.90$ was considered abnormal. ${ }^{15}$ Fisher's exact test and the Mann-Whitney $U$-test were applied. Logistic regression analysis was used to determine the odds of abnormal ABI using dichotomous predictors representing PEX and other systemic diseases (yes $=1$ and no $=0$ ).

\section{Results}

Of the 160 patients, 78 were females and 82 were males. The mean age of the patients was $68.83 \pm 4.62$ years in the controls and $69.0 \pm 5.82$ years in the cases (Table 1). In none of the subjects in the present study with PEX could we identify phacodonesis. There was no significant difference in lowest mean ABI between males and females within controls (males $v$ s females: $0.98 \pm 0.03$ vs $0.98 \pm 0.04 ; P=0.33$ ) and also within cases (males vs females: $0.89 \pm 0.03$ vs $0.88 \pm 0.03 ; P=0.06$ ). The lowest mean ABI documented in the controls was $0.98 \pm 0.03$ (range 0.86-1.08, median 0.97), whereas in the cases, it was $0.88 \pm 0.02$ (range $0.79-0.92$, median $0.89 ; P<0.001$ ). The odds ratio of abnormal ABI in cases as compared with controls was 58.50 (95\% CI 15.80-216.59).

The distribution of subjects in the two groups by systemic illnesses (HTN, DM and IHD, cerebrovascular stroke) was not statistically significant except for cerebrovascular stroke (Table 2). In the presence of systemic illness, the cases $(n=22)$ had a lower mean least $\mathrm{ABI} 0.88 \pm 0.02$ when compared with the controls $(n=45 ; 0.99 \pm 0.04) ; P<0.001$. Similarly, in the absence of systemic illness, the cases $(n=18)$ had a lower mean, least $\mathrm{ABI}(0.88 \pm 0.03)$ compared with the 75 controls (0.97 \pm 0.02$) ; P<0.001$ (Table 3). ABI was found to be significantly lower in cases as compared with controls irrespective of the state of systemic illness.

For the logistic regression analysis, patients were classified into two groups on the basis of the mean lowest $A B I$ value, namely normal $A B I$ and abnormal $A B I$. An $\mathrm{ABI}$ ratio of $<0.90$ was considered abnormal. This binary variable was taken as a dependent variable in order to detect the odds of falling into the abnormal ABI group.
Table 1 Distribution of patients in different age groups between controls (without PEX) and case (PEX)

\begin{tabular}{lccc}
\hline $\begin{array}{l}\text { Age group } \\
\text { (years) }\end{array}$ & $\begin{array}{c}\text { Controls } \\
\text { (without PEX; } \\
\mathrm{n}=\text { patients }(\%))\end{array}$ & $\begin{array}{c}\text { Case (PEX; } \\
\mathrm{n}=\text { patients } \\
(\%))\end{array}$ & $\begin{array}{c}\text { Total } \\
(\mathrm{n}=\text { patients } \\
(\%))\end{array}$ \\
\hline $60-64$ & $18(15)$ & $6(15)$ & $24(15)$ \\
$65-69$ & $51(42.5)$ & $17(42.5)$ & $68(42.5)$ \\
70 and above & $51(42.5)$ & $17(42.5)$ & $68(42.5)$ \\
\hline
\end{tabular}

Table 2 Distribution of proportion of patients with and without systemic illness between controls (without PEX) and cases (PEX)

\begin{tabular}{lrrr}
\hline & Absence & & Presence \\
\hline $\begin{array}{l}\text { Hypertension } \\
\quad \text { Control (\%) }\end{array}$ & $94(78.3)$ & & $26(21.7)$ \\
$\quad$ Case (\%) & $25(62.5)$ & & $15(37.5)$ \\
Fisher's exact test & & $P=0.06$ & \\
Diabetes mellitus & & & \\
$\quad$ Control (\%) & $98(81.7)$ & & $22(18.3)$ \\
$\quad$ Case (\%) & $30(75.0)$ & & $10(25.0)$ \\
Fisher's exact test & & $P=0.37$ & \\
Ischaemic heart disease & & & \\
$\quad$ Control (\%) & $102(85.0)$ & & $18(15.0)$ \\
$\quad$ Case (\%) & $33(82.5)$ & & $7(17.5)$ \\
Fisher's exact test & & $P=0.80$ & \\
Cerebrovascular stroke & & & \\
$\quad$ Control (\%) & $116(96.7)$ & & $4(3.3)$ \\
$\quad$ Case (\%) & $34(85.0)$ & & $6(15.0)$ \\
Fisher's exact test & & $P<0.01$ & \\
\hline
\end{tabular}

Table 3 Influence of the presence and absence of systemic illness on mean least ABI between controls (without PEX) and cases (PEX)

\begin{tabular}{lcccc}
\hline Group & $\mathrm{N}$ & Mean & Median & Mean rank \\
\hline $\begin{array}{l}\text { Presence of systemic illness } \\
\text { Control (without PEX) }\end{array}$ & 45 & $0.99 \pm 0.04$ & 0.98 & 44.19 \\
Case (PEX) & 22 & $0.88 \pm 0.02$ & 0.89 & 13.16 \\
& & & & \\
Absence of systemic illness & & & & \\
Control (without PEX) & 75 & $0.97 \pm 0.02$ & 0.97 & 55.83 \\
Case (PEX) & 18 & $0.89 \pm 0.03$ & 0.89 & 10.22 \\
\hline
\end{tabular}

Mann-Whitney $U$ test; $P<0.001$.

Dummy variables representing four systemic diseases (HT, DM, IHD, and cerebrovascular stroke), gender and PEX were taken as predictors. Table 4 shows the odds of belonging to the abnormal $\mathrm{ABI}$ group in the presence of HT, DM, IHD, cerebrovascular stroke, and PEX. Except for IHD, HT, gender, and PEX $(P<0.001)$, the other predictors did not attain statistical significance. Presence of HT and IHD increased the odds of abnormal ABI by 
Table 4 Outcomes of binary logistic regression analysis to determine the odds of abnormal ABI

\begin{tabular}{llr}
\hline Model & P-value & Odds \\
\hline Hypertension & $0.03^{*}$ & 5.58 \\
Diabetes mellitus & 0.84 & 1.19 \\
Ischemic heart disease & $0.03^{*}$ & 11.46 \\
Cerebrovascular stroke & 0.26 & 0.20 \\
Gender & $0.01^{*}$ & 6.68 \\
Pseudoexfoliation & $0.001^{*}$ & 151.05 \\
Constant & $0.001^{*}$ & 0.001 \\
\hline
\end{tabular}

Dependent variable: abnormal ABI.

*Statistical significance.

5 and 11 times, respectively. Females are six times more likely to have abnormal ABI compared with males. Whereas, after adjusting for other factors, in the presence of PEX the odds/chances of abnormal ABI was increased by 150 times. The logistic model achieved a high predictive accuracy of $91 \%$ and the Nagarkerke's $R$-square of 0.64 .

\section{Discussion}

Ocular PEX has been associated with cataract and glaucoma. Extraocular deposits of PEX have been localized to the connective tissues or septa traversing the organ tissue. These deposits are associated with the presence of elastic fibres, collagen fibres, fibroblasts, and the walls of small blood vessels, ${ }^{10}$ suggesting the systemic nature of PEX. An overexpression of the basic fibroblast growth factor, an imbalance in the matrix metalloproteinases (MMPs)/tissue inhibitors of MMPs, and increased cellular and oxidative stress, describe a part of the pathological process that is characterized by an elastic microfibrillopathy. ${ }^{16,17}$ The presence of fibrillopathy in the palpebral or bulbar conjunctiva and around the posterior ciliary vessels indicates that PEX was not just an intraocular disease ${ }^{10,11}$ and that it was a more diffuse process, which was demonstrated by the presence of similar material in the lid skin, orbital tissues, and other more remote areas of the skin and visceral organs. ${ }^{8-11}$ Further, it has been reported that in cases of established PEX, plasma homocysteine levels are elevated. ${ }^{18}$ Recent work suggests that PEX is a form of elastosis, and elastin is a major part of the ECM of arterioles. ${ }^{6,7,9,10,19}$ Recently, it has also been reported that there is a significant association between PEX and the DNA sequence variants in the gene coding for lysy-loxidase-like 1 , a protein responsible for elastin. ${ }^{20}$ PEX has been associated with transient ischaemic attacks, ${ }^{7}$ Alzheimer's disease, asymptomatic myocardial dysfunction, sensorineural hearing loss, stroke, myocardial infarction, systemic HT, and aneurysm of the abdominal aorta. ${ }^{9-12}$ However, to the best of our knowledge, the possible association between ocular PEX and PVD has not been reported. The purpose of this study was to investigate the association of PEX with PVD by measuring ABI.

Measurement of $\mathrm{ABI}$ is an essential investigation to identify patients with peripheral arterial disease, since clinical examination alone cannot exclude the presence of PVD. The ABI is the best 'non-invasive' test for PVD; and the measurement of $A B I$ is a sensitive and specific diagnostic test for PVD. ABI can be performed quickly and has high validity and good reproducibility. ${ }^{15,21}$ The importance of measuring ABI is also stressed by two additional facts. First, patients with asymptomatic PVD have similar vascular morbidity and mortality to patients with symptomatic PVD. Second, the prevalence of PVD is considerable; in population-based studies in Europe and the US, the prevalence of PVD (defined as ABI <0.9) was $\sim 5.8,10.9,12.2,18$ and $39 \%$ in individuals older than 40, 50, 60, 65 and 85 years, respectively. ${ }^{21}$ As per the US Preventive Services Task Force recommendations, testing for $\mathrm{ABI}$ is indicated in all symptomatic patients with PVD, and among asymptomatic patients at risk for PVD, such as in persons above age of 70 years, those aged 50-69 years, and those with a history of smoking or diabetes aged 70 years and older. Consequently, normative data for $\mathrm{ABI}$ and the cut-off have been derived for persons aged 50 years or above. From the above information, this value of ABI remains constant for all age group, or does not change according to age. ${ }^{21,22}$

In the present study, the mean least ABI value was lower in subjects with established PEX as compared with those without PEX. Further, the mean least ABI value was also lower in cases with established PEX in the presence as well as absence of systemic illness. Further, after adjusting for HT, DM, IHD, and CVS, logistic regression analysis demonstrated that the presence of PEX increased the odds of belonging to the abnormal ABI group by 150 times. This suggests a strong association between pseudoexfoliative material and PVD. PEX has also been shown to affect smaller vessels rather than the major ones. ${ }^{23}$ In few studies, it was suggested that co-existence of cerebrovascular disease, extra or intracranial, with abnormal iris transluminance, together with increased prevalence of PEX, further supported that hypoperfusion is a contributory factor in the development of PEX. ${ }^{24,25}$ Ringvold $^{26}$ has described PEX material in the blood vessel of the iris and conjunctiva, postulating that the process is not purely an intraocular one. In another study, authors found that the frequency of PEX was higher in the eyes of patients who have had transient ischaemic attacks compared with the eyes of healthy subjects of the same age, suggesting that hypoxia may have a contributory role in the development of 
PEX and was supported by the flourescein angiographic appearance of the iris in PEX. ${ }^{25}$ In the same study, the authors suggested that peripheral resistance of the ophthalmic artery resides in the ciliary and retinal circulations implying that the eyes of patients who had a PEX with positive iris transluminance have defective ocular circulation. ${ }^{25}$

In our previous study ${ }^{27}$ while evaluating intraoperative performance of Indian eyes with PEX, we found that the results were comparable to that in normal eyes in contrast to reports from other parts of world, which indicated more intraoperative difficulties and complications. From the current study, it would be justifiable to add that the results of current study may not be applicable to patient with PEX from other part of the world.

The clinical importance of this systemic manifestation is still unknown. Considering the above association, a slit-lamp examination of the eye could help in identifying an important marker that indicates the risk of a systemic vascular disease. Based on the results of current study, we strongly recommend evaluating for PVD in all subjects with PEX. To the best of our knowledge, this is the first reported association between PEX and low ABI, which indicates the presence of a PVD. The possible role of PEX as a risk factor or marker for PVD merits further investigation.

\section{Summary}

What was known before

- Pseudoexfoliation (PXE) is related to vascular disease, ie, cerebrovascular and cardiovascular disease.

What this study adds

- PXE is related to peripheral vascular disease.

\section{Conflict of interest}

The authors declare no conflict of interest.

\section{References}

1 Naumann GOH, Schlötzer-Schrehardt U, Küchle M. Pseudoexfoliation syndrome for the comprehensive ophthalmologist; intraocular and systemic manifestations. Ophthalmology 1998; 105: 951-968.

2 Bartholomew RS. Pseudoexfoliation and angle closure glaucoma. J Glaucoma 1981; 3: 213-216.

3 Moreno J, Duch S, Lajara J. Pseudoexfoliation syndrome: clinical factors related to capsular rupture in cataract surgery. Acta Ophthalmol 1993; 71: 181-184.

4 Alfaiate M, Leite E, Mira J, Cunha-Vaz JG. Prevalence and surgical complications of pseudoexfoliation syndrome in Portuguese patients with senile cataract. J Cataract Refract Surg 1996; 22: 972-976.
5 Skuta GL, Parrish II RK, Hodapp E, Forster RK, Rockwood EJ. Zonular dialysis during extracapsular cataract extraction in pseudoexfoliation syndrome. Arch Ophthalmol 1987; 105: 632-634.

6 Prince AM, Ritch R. Clinical signs of the pseudoexfoliation syndrome. Ophthalmology 1986; 93: 803-807.

7 Repo LP, Teräsvirta ME, Koivisto KJ. Generalized transluminance of the iris and the frequency of the pseudoexfoliation syndrome in the eyes of transient ischemic attack patients. Ophthalmology 1993; 100: 352-355.

8 Ritch R, Schlotzer-Schrehard U. Exfoliation (pseudoexfoliation) syndrome toward a new understanding. Acta Ophthalmol Scand 2001; 79: 213-217.

9 Streeten BW, Dark AJ, Wallace RN, Li YZ, Hoepner JA. Pseudoexfoliation fibrillopathy in the skin of patients with ocular pseudoexfoliation. Am J Ophthalmol 1990; 110: 490-499.

10 Scholotzer-Schrehardtz UM, Koca MR, Naumann GOH, Volkhol H. Pseudoexfoliation syndrome; ocular manifestation of a systemic disorder? Arch Ophthalmol 1992; 110: 1752-1756.

11 Streeten BW, Li ZY, Wallace RN, Eagle RC, Keshgegian AA. Pseudoexfoliation fibrillopathy in visceral organs of a patient with pseudoexfoliation syndrome. Arch Ophthalmol 1992; 110: 1757-1762.

12 Repo LP, Terasvirta ME, Tuovinen EJ. Generalized peripheral iris tran luminance in the pseudoexfoliation syndrome. Ophthalmology 1990; 97: 1027-1029.

13 Ruckley CV. Symptomatic and asymptomatic disease. In: Fowkes FGR (ed). Epidemiology of Peripheral Vascular Disease. Springer Verlag: London, UK, 1991, pp 127-140.

14 Newman AB, Siscowisk DS, Manolio TA, Polak J, Fried LP, Borhani NO et al. Ankle arm index as a marker of atherosclerosis in the cardiovascular health study. Circulation 1993; 88: 837-845.

15 Doobay AV, Anand SS. Sensitivity and specificity of the ankle bronchial index to predict future cardiovascular outcomes. a systemic review. Arterioscler Thromb Vasc Biol 2005; 22: 1463-1469.

16 Gartaganis SP, Georgakopoulos CD, Exarchou AM, Mela EK, Lamari F, Karamanos NK. Increased aqueous humor basic fibroblast growth factor and hyaluronan levels in relation to the exfoliation syndrome and exfoliative glaucoma. Acta Ophthalmol Scand 2001; 79: 572-575.

17 Gartaganis SP, Georgakopoulos CD, Mela EK, Exarchou A, Ziouti N, Assouti M et al. Matrix metalloproteinases and their inhibitors in exfoliation syndrome. Ophthalmic Res 2002; 34: 165-171.

18 Vasavada RM, Ritch R, Liebmann JM, Jole M. Plasma homocysteine is elevated in patients with exfoliation syndrome. Am J Ophthalmol 2003; 136: 41-46.

19 Netland PA, Ye H, Streeten BW, Hernandez MR. Elastosis of the lamina cribrosa in pseudoexfoliation syndrome with glaucoma. Ophthalmology 1995; 102: 878-886.

20 Thorlefsson G, Magnusson KP, Sulem P, Walters GB, Gudbjartsson DF, Stefansson $\mathrm{H}$ et al. Common sequence variants in the LOXL 1 gene confer susceptibility to exfoliation glaucoma. Science 2007; 317: 1397-1400.

21 Hirsch AT, Haskal ZJ, Hertzer NR, Bakal CW, Creager MA, Halperin JL et al. ACC/AHA 2005 Practice Guidelines for the management of patients with peripheral arterial disease (lower extremity, renal, mesenteric, and abdominal aortic): a collaborative report from the American Association for Vascular Surgery/Society for Vascular Surgery, Society for Cardiovascular Angiography and Interventions, Society for 
Vascular Medicine and Biology, Society of Interventional Radiology, and the ACC/AHA Task Force on Practice Guidelines (Writing Committee to Develop Guidelines for the Management of Patients With Peripheral Arterial Disease): endorsed by the American Association of Cardiovascular and Pulmonary Rehabilitation; National Heart, Lung, and Blood Institute; Society for Vascular Nursing; TransAtlantic Inter-Society Consensus; and Vascular Disease Foundation. Circulation 2006; 113: e463-e654.

22 Tziomalos K, Athyros VG, Karagiannis A, Mikhailidis DP. The role of ankle brachial index and carotid intima-media thickness in vascular risk stratification. Curr Opin Cardiol 2010; 25: 394-398.

23 Mitchell P, Wang JJ, Smith W. Association of pseudoexfoliation syndrome with increased vascular risk. Am J Ophthalmol 1997; 124: 685-687.
24 Shrum KR, Hattenhauer MG, Hodge D. Cardiovascular and cerebrovascular mortality associated with ocular pseudoexfoliation. Am J Ophthalmol 2000; 129: 83-86.

25 Repo LP, Suhonen MT, Teräsvirta ME, Koivisto KJ. Color Doppler imaging of the ophthalmic artery blood flow spectra of patients who have had a transient ischemic attack. Correlations with generalized iris transluminance and pseudoexfoliation syndrome. Ophthalmology 1995; 102: 1199-1205.

26 Ringvold A. Light and electron microscopy of the wall of iris vessels in eyes with and without exfoliation syndrome (pseudoexfoliation of the lens capsule). Virchows Arch A Pathol Pathol Anat 1970; 349: 1-9.

27 Shastri L, Vasavada A. Phacoemulsification in Indian eyes with pseudoexfoliation syndrome. J Cataract Refract Surg 2001; 27: 1629-1637. 